\title{
El papel del ácido gamma-aminobutírico en la depresión de la mujer
}

\section{Mónica Flores-Ramos 1,2 , Margus Salinas 3 , Armando Carvajal-Lohr y Lorena Rodríguez-Bores²}

${ }^{1}$ Catedrática del Consejo Nacional de Ciencia y Tecnología; ${ }^{2}$ Clínica de Trastornos Afectivos, Instituto Nacional de Psiquiatría Ramón de la Fuente Muñiz; ${ }^{3}$ Facultad de Medicina, Universidad Nacional Autónoma de México. Ciudad de México; ${ }^{4}$ Departamento de Ciencias Biológicas y de la Salud, Universidad de Sonora, Hermosillo, Son. México

\section{Resumen}

La depresión es un trastorno psiquiátrico frecuente e incapacitante. Es causada por diferentes factores, entre los que figura el aspecto neurobiológico. En el presente trabajo presentamos evidencias acerca del papel del ácido gamma-aminobutírico (GABA) en la etiopatogenia de la depresión, con énfasis en la mujer. Los estudios en animales fundamentan la implicación del GABA en la depresión, mientras que los estudios clínicos han demostrado que el GABA se encuentra disminuido en líquido cefalorraquídeo (LCR) y plasma en pacientes con depresión. Estudios con espectroscopia muestran una disminución del GABA en diferentes áreas cerebrales. Tras la administración de antidepresivos, se incrementa y se observa mejoría clínica. Se ha visto que la depresión se presenta con mayor frecuencia en las mujeres que en los hombres, y se ha sugerido que existe una relación entre la depresión y los cambios hormonales. De igual manera, se ha visto que las hormonas gonadales tienen un efecto regulador del estrés sobre el eje hipotálamo-hipófisis-adrenal mediante receptores GABAérgicos, haciendo a las mujeres más vulnerables a sufrir estrés y por tanto depresión. Por lo anterior, se propone estudiar con mayor profundidad la implicación del GABA en la depresión, para buscar nuevas estrategias terapéuticas.

PALABRAS CLAVE: GABA. Depresión mayor. Mujer. Imágenes por resonancia magnética. Espectroscopía. Hormonas.

\begin{abstract}
Depression is a common psychiatric disorder and a leading cause of disability worldwide. Multiple and diverse factors are involved in its cause although biologic factors are prominent. The present study reviews the evidence about the role that gamma-aminobutyric acid plays in the complex pathogenesis of depression, particularly in women. The implication of gamma-aminobutyric acid (GABA) is based mainly from animal models whereas clinical studies in depressed patients show alterations of GABA levels in plasma and cerebrospinal fluid. Neuroimaging studies using spectroscopy indicate also decreased $G A B A$ levels in different brain areas which in turn may normalize after antidepressant therapy, and these findings translate into clinical response. It has been observed that depression has a higher prevalence among women which suggests a link between depression and hormonal changes. Similarly, gonadal hormones have a regulatory effect on the hypothalamic-pituitary-adrenal axis through GABA receptors making women more vulnerable to suffer stress and depression. Therefore, the implication of GABA in the neurobiology of depression should be explored in order to search for new therapeutic strategies.
\end{abstract}

KEY WORDS: GABA. Major depression. Women. Magnetic resonance imaging. Spectroscopy. Hormones.

\author{
Correspondencia: \\ Mónica Flores-Ramos \\ Instituto Nacional de Psiquiatría Ramón de la \\ Fuente Muñiz \\ Calzada México-Xochimilco, 101 \\ C.P. 14370, Huipulco, Ciudad de México, México \\ E-mail: flores_ramos@hotmail.com
}

Fecha de recepción en versión modificada: 27-04-2016

Fecha de aceptación: 30-04-2016

DOI:10.24875/GMM.17002544
Gac Med Mex. 2017;153:486-495

Contents available at PubMed

www.gacetamedicademexico.com 


\section{Introducción}

En octubre de 2012, la Organización Mundial de la Salud (OMS) emitió un reporte donde destacaba el TDM como una enfermedad altamente prevalente, presente en más de 350 millones de personas en el mundo, considerándolo como la principal causa de discapacidad y un factor que contribuye de manera importante a la morbimortalidad. Por lo anterior, la OMS ha considerado la atención de este trastorno como un programa prioritario de salud 1 .

La depresión tiene un origen multifactorial, en el cual inciden una serie de factores como el sexo, enfermedades médicas asociadas, aislamiento social, deterioro cognoscitivo, eventos estresantes, antecedentes personales y familiares de trastorno depresivo mayor (TDM), nivel socioeconómico bajo, dolor crónico y trastorno del sueño, y factores neurobiológicos, entre otros ${ }^{2}$.

En el aspecto neurobiológico se ha considerado que alteraciones en los neurotransmisores pueden ser la causa de los cambios anímicos ${ }^{3}$. La hipótesis monoaminérgica de la depresión ha sido el eje a considerar en la etiología de la depresión; sin embargo, también se ha observado un papel importante de otros sistemas de neurotransmisión en la génesis del trastorno, entre ellos el del ácido gamma-aminobutírico (GABA).

Presentamos evidencias acerca del papel que el sistema GABAérgico desempeña en la etiopatogenia de la depresión mayor, con énfasis en la depresión del sexo femenino, que tiene características específicas, las cuales se mencionarán en el texto.

\section{Aspectos básicos del ácido gamma-aminobutírico}

El GABA es el principal neurotransmisor inhibidor del sistema nervioso central (SNC) ${ }^{4}$ y modula el balance inhibitorio-excitatorio en el cerebro maduro ${ }^{5}$. Se involucra aproximadamente en un tercio de las sinapsis en regiones como la corteza cerebral, el hipocampo, el tálamo, los ganglios basales, el cerebelo, el hipotálamo y el tallo cerebral6-8, y en circuitos interneuronales como los noradrenérgicos, dopaminérgicos y serotoninérgicos ${ }^{9}$.

\section{Metabolismo del ácido gamma-aminobutírico}

En las terminales GABAérgicas, el GABA se forma a partir del glutamato por la reacción enzimática mediada por la ácido glutámico descarboxilasa (GAD), utilizando el fosfato de piridoxal como cofactor $^{10}$. Los transportadores del GABA, que se encuentran distribuidos por diferentes lugares del SNC (Tabla 1), se encargan de recaptar el GABA extracelular desde las terminales presinápticas, ya que este neurotransmisor no está sujeto a degradación enzimática ${ }^{11}$.

El GABA que es captado por los astrocitos no se utiliza de primera instancia, ya que es metabolizado a semialdehído succínico (SSA) por la GABA-transaminasa. A su vez, el SSA es oxidado por la SSA deshidrogenasa (SSA-DH) a ácido succínico, el cual entra de nuevo al ciclo de Krebs para ser transformado en glutamato o en ácido gamma-hidroxibutírico por la aldehído reductasa9.

El glutamato, en los astrocitos, se transforma en glutamina por la glutamina sintetasa para posteriormente ser transportado a los axones terminales. En las terminaciones nerviosas, la glutamina es convertida en glutamato por la enzima glutaminasa; posteriormente, la GAD transforma el glutamato en GABA ${ }^{10-12}$. El GABA es captado por los transportadores neuronales para liberarse, ya que las terminaciones nerviosas contienen GAD o en ellas se encuentran los transportadores para la recaptura del GABA hacia las vesículas sinápticas ${ }^{13}$ (Fig. 1).

\section{Receptores del ácido gamma-aminobutírico}

Existen dos tipos de receptores GABAérgicos: el receptor ionotrópico $\mathrm{GABA}_{A}$ y el receptor metabotrópico $\mathrm{GABA}_{B}{ }^{5}$. En el cerebro adulto, el GABA actúa principalmente por la activación de los receptores $\mathrm{GABA}_{\mathrm{A}}{ }^{14}$.

Los receptores $\mathrm{GABA}_{A}$ son ionotrópicos y en su mayoría postsinápticos localizados en la parte apical de las dendritas de las neuronas, ocasionando un rápido potencial postsináptico inhibitorio ${ }^{15}$. Son receptores heteroligoméricos organizados en un canal compuesto de cinco subunidades: $\alpha 1-\alpha 6, \beta 1-\beta 4, \Upsilon 1-y 3$, e, $\ominus, \pi$ у $1-p 3)^{16}$.

Los receptores $\mathrm{GABA}_{\mathrm{B}}$ median su acción inhibitoria a través de sistemas de segundos mensajeros

\begin{tabular}{l}
$\begin{array}{l}\text { Tabla 1. Transportadores del GABA y su principal distribución en } \\
\text { el sistema nervioso central }\end{array}$ \\
$\begin{array}{ll}\text { GAT-1 } & \begin{array}{l}\text { Cerebelo, ganglios basales, bulbo olfatorio, retina y } \\
\text { núcleo interpeduncular }\end{array} \\
\text { GAT-2 } & \begin{array}{l}\text { Leptomeninges, epéndimo y células del plexo } \\
\text { coroideo }\end{array} \\
\text { GAT-3 } & \text { Bulbo olfatorio y retina }\end{array}$ \\
\hline
\end{tabular}




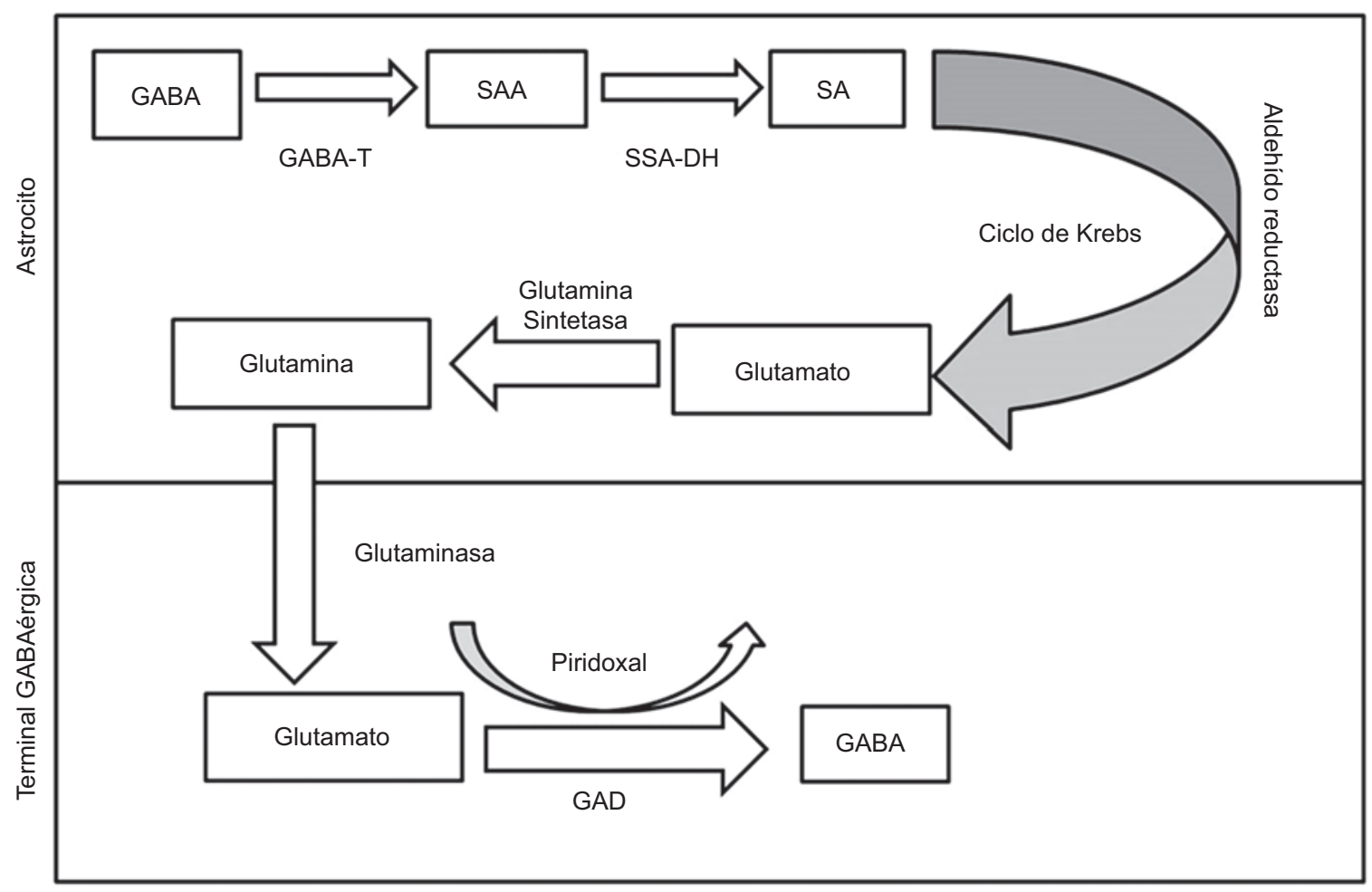

Figura 1. Metabolismo del GABA. GABA-T: GABA transaminasa; GAD: ácido glutámico descarboxilasa; SA: ácido succínico; SAA: semialdehído succínico; SSA-DH: SSA deshidrogenasa.

acoplados a la proteína $\mathrm{G}$ que regulan la liberación de neurotransmisores y la actividad de los canales iónicos y de la adenilciclasa, resultando en una respuesta más lenta y tardía del potencial inhibitorio $^{17,18}$. Están compuestos por dos subunidades: GA$\mathrm{BA}_{\mathrm{B} 1}$ (más abundante en el periodo natal y asociado a estructuras presinápticas) ${ }^{19}$ y $\mathrm{GABA}_{\mathrm{B} 2}$ (más abundante en el cerebro adulto y asociado a estructuras postsinápticas) $)^{14,19}$.

\section{Papel de los receptores del ácido gamma- aminobutírico en la depresión}

\section{Receptor $\mathrm{GABA}_{A}$}

El déficit del receptor $\mathrm{GABA}_{A}$ se encuentra implicado en diversos trastornos neuropsiquiátricos, entre los que se encuentra la depresión. Se han realizado estudios para dilucidar la relación de este receptor con la depresión, especialmente con aquella de características ansiosas. Por ahora sabemos que el receptor GABA tiene un papel esencial en el desarrollo del $\mathrm{SNC}^{20}$, en la regulación de la respuesta asociada al estrés a largo plazo ${ }^{21}$ y en la acción que ejercen diversos antidepresivos y estabilizadores del ánimo, que es mediada por este receptor ${ }^{22}$. Diversos estudios clínicos y en modelos animales han evaluado la relación entre las subunidades del receptor $\mathrm{GABA}_{A}$ y la depresión.

En estudios de nado forzado en ratas, la deleción de la subunidad $\gamma 2$ ha tenido como resultado la inmovilidad de los animales, sugiriendo una probable relación con la depresión. Se han encontrado diversas funciones de esta subunidad, entre las que destaca su relación con la modulación de la proliferación, maduración e integración de las vías sinápticas de las neuronas en el hipocampo, por lo que su déficit se relaciona con alteraciones de las mismas, lo que puede ser modificado con la administración crónica de antidepresivos durante la vida adulta ${ }^{23}$.

Otra de las relaciones importantes de la subunidad $\gamma 2$ es aquella que comparte con el eje hipotalámico-hipofisiario-adrenal (HHA). Los estudios demuestran que los antidepresivos tricíclicos, al normalizar la subunidad $\gamma 2$, estabilizan la hiperfunción del eje HHA, lo que se traduce en una mejoría de los síntomas de la depresión con características ansiosas ${ }^{24}$.

En cuanto a la subunidad $\delta$, se ha propuesto que desempeña un papel importante en la depresión posparto. Se ha observado que el incremento de la 
progesterona (propio del embarazo) ejerce una disminución de la expresión de las subunidades $\gamma_{2}$ y $\delta$, reflejándose en una depresión posparto de características ansiosas ${ }^{24}$. Sin embargo, se ha encontrado que las alteraciones en el equilibrio de los neuroesteroides alopregnanolona e isopregnanolona pueden ser corregidas con la administración de inhibidores selectivos de la recaptura de serotonina (ISRS) ${ }^{25}$.

Desgraciadamente, aún nos encontramos lejos de descubrir por completo los efectos del receptor $\mathrm{GABA}_{\mathrm{A}}$ en la etiología de la depresión, pero diversos estudios aportan evidencia de la importante relación entre estos receptores y los mecanismos de neurotransmisión involucrados en la depresión. Por ejemplo: la asociación de la subunidad $\alpha_{3}$ con la alteración de los sistemas dopaminérgicos, serotoninérgicos y noradrenérgicos ${ }^{26}$; el hecho de que la subunidad $\alpha_{2}$ está altamente expresada en la amígdala, el hipocampo y el núcleo accumbens, que son objeto de interés para el estudio del estrés ${ }^{27}$; y por último, la ubicación de la subunidad $\beta$ en el giro dentado del hipocampo, cuya expresión se ve aumentada con el consumo crónico de estabilizadores del ánimo ${ }^{28}$.

\section{Receptor $\mathrm{GABA}_{B}$}

Aún no se encuentra del todo establecida la función del receptor $\mathrm{GABA}_{B}$ en la génesis de los trastornos afectivos ${ }^{29}$. Estudios realizados en animales sugieren que los agonistas de estos receptores poseen actividad de tipo antidepresivo ${ }^{30}$. Sin embargo, esta premisa puede ser debatida con el estudio de Nakagawa, et al. $^{31}$, quienes observaron en un modelo de desesperanza aprendida que el incremento a largo plazo de la neurotransmisión de $\mathrm{GABA}_{\mathrm{B}}$ estaba relacionado con la desesperanza en ratas.

Estudios recientes han demostrado que los antagonistas del receptor $\mathrm{GABA}_{B}$ muestran un efecto neuroprotector, siendo capaces de producir un rápido incremento del factor neurotrófico derivado del cerebro y del factor de crecimiento nervioso ${ }^{32}$. Se ha encontrado también que los antidepresivos, al igual que los antagonistas del receptor $\mathrm{GABA}_{B}$, aumentan las concentraciones de los factores antes mencionados, lo que sugiere que ambos tienen un efecto de atenuación del proceso neurodegenerativo presente en la depresión ${ }^{33-35}$.

El agotamiento de la concentración de serotonina en el cerebro bloquea los efectos antidepresivos de los antagonistas del GABA ${ }_{B}{ }^{36}$. Además, los receptores de serotonina se encuentras acoplados a los mismos canales de potasio que el receptor $\mathrm{GABA}_{\mathrm{B}}{ }^{37}$; otra evidencia más de que el sistema GABAérgico ejerce un importante papel en la modulación del estado de ánimo.

Gracias al avance en los estudios de imágenes cerebrales ha sido posible encontrar las zonas con mayores concentraciones del receptor $\mathrm{GABA}_{B}$, entre las que destacan la capa molecular del cerebelo, el núcleo interpeduncular, la corteza frontal, el núcleo anterior olfatorio y el núcleo del tálamo ${ }^{38}$. Además, se ha observado que el tratamiento crónico con antidepresivos se refleja en una gran presencia del receptor $\mathrm{GABA}_{\mathrm{B}}{ }^{39}$.

\section{Estudios clínicos: trastorno depresivo mayor y ácido gamma-aminobutírico}

En 1980, Emrich sugirió por primera vez que el neurotransmisor GABA se encontraba implicado en la modulación del afecto, a partir de la observación de que el valproato (un compuesto GABAmimético) presentaba un efecto antimaniaco ${ }^{40}$. Estudios posteriores continuaron aportando evidencia sobre el vínculo del GABA con el trastorno depresivo. Gold, et al. ${ }^{41}$ observaron que la concentración de GABA en el LCR de pacientes deprimidos se encontraba significativamente reducida, en comparación con pacientes con enfermedad neurológica ${ }^{41}$. Sin embargo, una limitación importante observada en su trabajo fue la imposibilidad técnica para determinar el sitio de procedencia del GABA presente en el LCR y la imposibilidad de establecer una relación entre los valores del GABA en el LCR y en determinadas áreas cerebrales.

Mann, et al. ${ }^{42}$ llevaron a cabo un estudio en el cual compararon los valores de GABA en el LCR en 167 pacientes (130 diagnosticados con TDM y 37 con depresión bipolar según los criterios del DSM-IV) y 38 voluntarios sanos evaluados con la escala de Hamilton de depresión. Las puntuaciones basales de los pacientes con alguno de los dos trastornos afectivos fueron de $20 \pm 6.1$, mientras que las de los controles sanos fueron de $0.6 \pm 0.9$. Se encontró que los valores del GABA en el LCR fueron menores en los pacientes con TDM que en los voluntarios sanos y en aquellos con depresión bipolar; no se encontró correlación entre los valores del GABA en el LCR y la gravedad de la depresión en la escala de Hamilton $(p=0.308)$. Por lo tanto, podemos concluir que los valores GABAérgicos se relacionan con la presencia de depresión unipolar, pero no con la gravedad del cuadro depresivo. Por otro lado, existen estudios que 
indican que los valores del GABA en el LCR pueden tener relación con la presencia de ciertos síntomas propios de la depresión, como la anhedonia y las tendencias suicidas ${ }^{43}$.

Otra evidencia indirecta de la participación del sistema GABAérgico en la depresión proviene de ensayos clínicos que demuestran las propiedades antidepresivas de compuestos GABAmiméticos ${ }^{44-46}$. En la revisión realizada por Van Marwijk, et al. ${ }^{44}$ se analizaron 21 estudios que utilizaban alprazolam como monoterapia para el TDM en comparación con placebo y con antidepresivos convencionales. Los autores encuentran que el alprazolam, al compararlo con placebo, reduce la sintomatología depresiva, y con respecto a los antidepresivos tricíclicos es igual de efectivo como monoterapia ${ }^{44}$. Concluyen que, a pesar de que la evidencia indica que efectivamente el sistema GABAérgico participa en la depresión y que los compuestos GABAmiméticos demuestran propiedades antidepresivas, se debe tomar con precuación el uso de benzodiacepinas por el potencial desarrollo de tolerancia.

La evaluación del GABA también se ha realizado en plasma. Un estudio analizó las diferencias en las concentraciones plasmáticas de GABA entre pacientes con depresión y mujeres sanas; además, evaluó los cambios en dichas concentraciones después de la administración de fluoxetina o escitalopram durante 10 días. Los resultados mostraron que las concentraciones sanguíneas de GABA eran menores en las mujeres deprimidas que en las controles. En las mujeres bajo tratamiento, se observó que las concentraciones plasmáticas de GABA se incrementaron con respecto a las basales ${ }^{47}$.

\section{Estudios de espectroscopía en pacientes con depresión}

El estudio del papel de GABA en la etiopatogenia de la depresión retomó importancia con el surgimiento de las técnicas de imágenes cerebrales no invasivas, como la espectroscopía de protones por imágenes de resonancia magnética que permite la evaluación de los metabolitos en el cerebro. En un estudio realizado por Sanacora, et al. ${ }^{48}$ se analizó a 11 sujetos con diagnóstico de TDM, según los criterios del DSM-IV, que se encontraban sin tratamiento con psicofármacos. Se les realizó una resonancia magnética de protones con espectroscopía para determinar los valores del GABA en la corteza occipital. Posterior a este estudio, se les administró ISRS como monoterapia (fluoxetina o escitalopram) durante 5 semanas. Se observó que las concentraciones de GABA aumentaron hasta un $34 \%$ en la corteza occipital. Esto sugiere que los ISRS estimulan los receptores de serotonina $5 \mathrm{HT}_{3} \circ 5 \mathrm{HT}_{2 \mathrm{~A}}$, que se encuentran en las neuronas GABAérgicas, favoreciendo la liberación de GABA.

Por medio de estudios de espectroscopía se logró comprobar la existencia de valores bajos de GABA en pacientes con TDM y depresión resistente al tratamiento. En un estudio comparativo realizado en pacientes con depresión resistente al tratamiento, sujetos sanos y pacientes respondedores al tratamiento se encontró que los pacientes con depresión resistente presentaron menores concentraciones de GABA en la corteza occipital y la corteza anterior del cíngulo ${ }^{49}$.

Las áreas cerebrales que se han reportado con mayor frecuencia con concentraciones disminuidas de GABA son la corteza occipital, la corteza anterior del cíngulo y las regiones dorsomedial y dorsal anterolateral de la corteza prefrontral ${ }^{50,51}$. El hallazgo de concentraciones disminuidas del neurotransmisor GABA en la corteza occipital ha podido replicarse en diversos estudios; sin embargo, se ha propuesto centrar más la atención en la corteza del cíngulo y la corteza prefrontal, debido a que estas áreas anatómicas tienen una conocida implicación en la regulación del estado de ánimo ${ }^{52}$. Los estudios de imagen también han comenzado a utilizarse para medir la respuesta al tratamiento y establecer el pronóstico en los pacientes con depresión. Existen estudios que reportan un incremento del GABA en la corteza occipital después de un tratamiento antidepresivo efectivo con terapia electroconvulsiva ${ }^{53}$, y estudios que reportan concentraciones GABAérgicas normales en pacientes deprimidos recuperados y sin medicación ${ }^{54}$.

El estudio de la depresión, como hemos mencionado con anterioridad, implica una correlación multidisciplinaria para poder desentrañar la compleja gama de causas que la integran, por lo que en un intento de continuar dilucidando la relación del GABA con la depresión se han realizado estudios post mortem que reportan una reducción en el tamaño y la densidad de las neuronas en la corteza prefrontal dorsolateral en pacientes con depresión mayor, relacionándolo con la reducción de las concentraciones corticales de GABA ${ }^{55}$.

\section{La depresión en la mujer}

Se sabe que el TDM se presenta con mayor frecuencia en las mujeres que en los hombres, en una 
proporción de 2:156. Esta diferencia se hace más evidente durante la etapa fértil de la vida de la mujer ${ }^{56,57}$. Sin embargo, ciertas etapas reproductivas, como la fase premenstrual, el posparto y la perimenopausia, se ven acompañadas de sintomatología depresiva en mujeres vulnerables, lo cual se supone que se debe a su estrecha relación con cambios hormonales ${ }^{57,58}$. De igual manera, parece existir una relación entre la presencia de cuadros depresivos en la perimenopausia con eventos previos de depresión, trastorno disfórico premenstrual y depresión posparto ${ }^{59}$. Lo anterior sugiere que las hormonas gonadales deben tener algún papel en la vulnerabilidad para sufrir episodios depresivos y en las diferencias de sexo de la presentación de la depresión.

Las mediciones séricas de hormonas gonadales que se han llevado a cabo en mujeres que sufren trastornos afectivos y se encuentran en diferentes etapas reproductivas han arrojado resultados diversos, y en ocasiones contradictorios. Freeman, et al. ${ }^{60}$ observaron que las concentraciones crecientes de hormona estimulante del folículo (FSH) y de hormona luteinizante, así como la fluctuación en los valores de estradiol, se relacionaban con altas puntuaciones en las escalas de medición de la depresión, aun después de realizar un análisis con control de variables clínicas y psicosociales. De manera similar, se ha observado que es el incremento de la FSH y la disminución del estradiol, pero no las concentraciones séricas de estos en un momento dado, lo que se encuentra relacionado con los síntomas depresivos en las mujeres posmenopáusicas ${ }^{61}$. Más aún, la mejoría en los síntomas depresivos en las mujeres en la perimenopausia se relaciona de manera estrecha con la disminución en las concentraciones séricas de $\mathrm{FSH}^{62}$.

Por otro lado, también se ha observado que la testosterona puede estar relacionada con la presencia de síntomas depresivos en mujeres que transitan hacia la menopausia. Bromberger, et al. ${ }^{63}$, en 2010, reportaron un trabajo de seguimiento de 3302 mujeres entre 42 y 52 años de edad, inicialmente premenopáusicas, a quienes midieron las concentraciones séricas de estradiol, testosterona, FSH, globulina fijadora de hormonas sexuales y sulfato de dehidroepiandrosterona cada año durante ocho años. Observaron una relación entre las concentraciones séricas de testosterona y la presencia de síntomas depresivos, pero no con el resto de las hormonas evaluadas.

Así como la presencia o la ausencia de depresión se han relacionado con diferentes hormonas, también existe registro de la relación entre hormonas y síntomas específicos de la depresión. Las concentraciones séricas de testosterona se relacionan con la presencia de insomnio durante la transición a la menopausia ${ }^{64}$.

Se necesita más evidencia para lograr un consenso en cuanto a qué papel representan las hormonas gonadales en la presencia de síntomas afectivos durante diferentes etapas de la vida, como la transición a la menopausia ${ }^{65}$, el posparto ${ }^{66}$ y la vida fértil ${ }^{67}$. Sin embargo, una probable explicación para este fenómeno podría derivarse de las diferencias de la regulación del estrés entre mujeres y hombres, lo cual se encuentra relacionado con la participación de las hormonas gonadales ${ }^{68}$. El estrés es regulado por una compleja red de hormonas y neurotransmisores que preparan al organismo para atender las demandas de cambios ambientales y regresarlo a la homeostasis. El mecanismo con mayor peso en este proceso es el que realiza el eje HHA como regulador de los moduladores del estrés. De acuerdo con ciertas investigaciones, se ha propuesto que los estrógenos facilitan la actividad del eje HHA, mientras que la progesterona y la testosterona reducen la actividad del eje encargado del estrés ${ }^{69}$. Sin embargo, existen ciertos metabolitos de estas hormonas que, al interaccionar con receptores de estrógenos, facilitan la actividad del eje del estrés ${ }^{70}$. Por ende, nos encontramos ante un delicado balance entre los efectos de activación o inhibición que ejercen las hormonas y sus derivados sobre este importante eje $\mathrm{e}^{71}$. Aparentemente, los efectos de las hormonas sobre el eje están mediados por receptores GABAérgicos ${ }^{68}$.

Como ya hemos mencionado, existen diversos trabajos que reportan los efectos de las hormonas gonadales femeninas en el estado de ánimo. Claros ejemplos son el impacto de la regulación que ejerce el estradiol sobre la actividad cerebral y la plasticidad del mismo, los numerosos estudios en modelos animales que sustentan el efecto del estradiol como antidepresivo en modelos animales con menopausia $^{72-74}$ y los trabajos sobre población clínica que demuestran los efectos benéficos que los estrógenos tienen sobre el ánimo ${ }^{75-77}$. De igual forma, se han encontrado efectos antidepresivos en la dehidroepiandrosterona ${ }^{78}$ y la progesterona con su metabolito $5 \alpha$-reducido, alopregnanolona, que producen efectos antidepresivos ${ }^{79}$, al parecer a través de su unión con el receptor $\mathrm{GABA}_{\mathrm{A}}{ }^{80}$.

En el caso de otras hormonas, como la progesterona y la alopregnanolona, se ha estudiado su 
relación con otros síndromes relacionados como la depresión; tal es el caso del trastorno disfórico premenstrual. Schmidt, et al. ${ }^{81}$ demostraron que los síntomas de esta afección pueden ser bloqueados cuando se inhibe el ciclo menstrual; además, demostraron que la adición de estradiol y progesterona en las mujeres que padecían este trastorno ocasionaba un incremento en el ánimo negativo, evento que no ocurría en aquellas mujeres que no presentaban este síndrome. Esto se explica con el hecho de que durante la fase lútea existe una mayor concentración de progesterona y de sus metabolitos, por lo que ejercen cambios en la modulación de los receptores $\mathrm{GABA}_{A}{ }^{50}$. Es importante mencionar que las pacientes con trastorno disfórico premenstrual han presentado un incremento de la excitabilidad de la corteza motora durante la fase lútea, cuando los valores de la progesterona están ligeramente elevados, en comparación con las controles, quienes presentaron una excitabilidad reducida $^{82}$. De ello se deduce que el efecto que producen estas hormonas sobre el estado de ánimo y los trastornos del afecto se encuentran ligados a los efectos que producen sobre la actividad del sistema GABAérgico, que consisten en modular la apertura del canal de cloro acoplado a dicho receptor y, por lo tanto, modificar la polaridad de la neurona. Debido a esto, un aumento en el tono GABAérgico localizado principalmente en los núcleos paraventriculares del hipotálamo se traduce en una disminución de la actividad en el eje HHA, y en consecuencia nos habla de una región crítica en lo que se refiere al control del estrés $^{83}$.

Así pues, las fluctuaciones en las hormonas ováricas pueden alterar la regulación del eje HHA mediada por GABA; tal es el caso de las mujeres con fallas en los receptores GABAérgicos, induciendo la disfunción del eje HHA y teniendo como resultado un incremento en la vulnerabilidad al estrés $y$, por lo tanto, mayor probabilidad de padecer depresión ${ }^{84}$.

Esta premisa ha dado pauta a múltiples investigaciones que tienen como objetivo evaluar las concentraciones de GABA en las mujeres durante periodos reproductivos específicos, como la fase lútea, el periodo perinatal y la perimenopausia, concluyendo que la función GABAérgica fluctúa a través del ciclo menstrual y evidenciando una clara diferencia entre mujeres con trastornos afectivos y mujeres sin estas afectaciones. Ahondando más en esto, una revisión sugiere que existe un gradiente de inhibición del GABA que difiere entre las mujeres con y sin depresión, de forma que aquellas con depresión presentarán una mayor inhibición durante la fase folicular media, la cual disminuye durante la fase lútea y llega a sus niveles más bajos durante la menstruación ${ }^{85}$. Este gradiente parece coincidir con el gradiente de la sintomatología depresiva posterior a la ovulación.

\section{Implicaciones clínicas}

El correlato clínico de la regulación del sistema GABAérgico en la depresión incluye aspectos como la predisposición a la enfermedad (vulnerabilidad al estrés), el curso de la misma y el tratamiento. Particularmente en la mujer, el curso de la depresión puede verse afectado por los ciclos reproductivos, que como se mencionó previamente coinciden con la inhibición de GABA mediada por las hormonas gonadales ${ }^{85}$. En relación al tratamiento de la depresión, se ha sugerido que las benzodiacepinas tienen un efecto antidepresivo por sí mismas a través de su acción sobre los receptores $\mathrm{GABA}_{A}{ }^{86}$. Particularmente el alprazolam ha sido estudiado en este sentido, y se ha visto relacionado con una disminución de los síntomas propios de la depresión, como la anhedonia y la desesperanza. Además, parece tener un rápido inicio de acción en comparación con los antidepresivos tricíclicos $^{46}$. Una revisión sistemática que incluyó 21 estudios, con un total de 2,693 participantes, y que tuvo como objetivo evaluar el efecto del alprazolam sobre los síntomas depresivos, reportó que este fármaco reduce los síntomas depresivos en mayor medida que el placebo y que es igualmente efectivo que los antidepresivos tricíclicos ${ }^{44}$.

Otro fármaco cuyo mecanismo de acción implica la regulación de los receptores GABAérgicos es el valproato, que tiene como efecto final un incremento en la concentración de GABA en el SNC por distintos mecanismos ${ }^{87}$, y que por tal motivo podríamos considerar útil para el tratamiento de la depresión. Si bien este fármaco es utilizado como estabilizador del estado de ánimo en pacientes con trastorno bipolar, existen algunos estudios que han evaluado su potencial antidepresivo, ya sea como monoterapia o junto a otros antidepresivos. Davis, et al. ${ }^{88}$ realizaron un estudio en 33 pacientes con TDM sin antecedentes de sintomatología maníaca o hipomaníaca durante ocho semanas en un ensayo abierto con valproato. Al término del estudio, el $66 \%$ de la muestra fueron respondedores y la media total de la puntuación de la escala de depresión de Hamilton disminuyó un $55 \%$. Los resultados sugieren que el valproato pudiera ser un tratamiento 
efectivo para el TDM, pero tenemos que considerar las limitaciones metodológicas que un estudio abierto conlleva. Igualmente se ha observado que los pacientes se ven beneficiados en su sintomatología depresiva cuando se agrega valproato como terapia adjunta a los ISRS $^{89}$ o como coadyuvante de la terapia electroconvulsiva ${ }^{90}$. Por otro lado, se ha visto que agregar valproato al tratamiento usual de pacientes con depresión resistente al tratamiento provee una mejoría clínica sustancial y un periodo de mantenimiento relativamente largo en este subgrupo de pacientes ${ }^{91}$.

Si bien los resultados de los estudios que evalúan al valproato como terapia única para la depresión no son concluyentes, y el uso de benzodiacepinas se ve restringido por su potencial adictivo, es importante considerar como terapia antidepresiva única o coadyuvante otras alternativas terapéuticas con mecanismo de acción sobre las vías gabaérgicas. Es el caso de la estimulación magnética transcraneal repetitiva, que ha demostrado ser efectiva para el tratamiento de la depresión y que se encuentra implicada en la modulación de los sistemas GABA/glutamato ${ }^{92}$, y de los fármacos que modulan el mismo sistema, como la ketamina ${ }^{92}$ y el zolpi$\mathrm{dem}^{93}$. De igual manera, evaluar el potencial terapéutico antidepresivo de esteroides neuroactivos ${ }^{94}$ podría permitir la propuesta de estrategias terapéuticas novedosas para pacientes con depresión o para grupos particulares de pacientes deprimidos, como podrían ser las mujeres con depresión posparto o durante la perimenopausia y los pacientes con depresión resistente al tratamiento.

\section{Conclusión}

Existe evidencia suficiente para considerar que el sistema GABAérgico desempeña un papel importante en la etiopatogenia de la depresión. Tanto los estudios en animales como las aportaciones clínicas coinciden en la importancia que dicho sistema tiene sobre la presencia de síntomas depresivos. Los estudios de neuroimagen aportan evidencia adicional, y particularmente el uso de espectroscopía permite evaluar los metabolitos cerebrales. En este tipo de estudios, el hallazgo predominante en pacientes deprimidos ha sido la disminución GABAérgica en la corteza occipital, la corteza anterior del cíngulo y la corteza prefrontal. En la mujer, el sistema GABAérgico parece cobrar importancia en cuanto a la modulación del estrés. Se propone que las hormonas gonadales tendrían un efecto sobre el eje HHA, mediado por receptores GABAérgicos, haciendo a las mujeres más vulnerables al estrés $y$, por tanto, más propensas a sufrir depresión.
Los hallazgos presentados en el presente trabajo sugieren anormalidades en el ciclo metabólico glutamato/glutamina/GABA en áreas cerebrales clave en la fisiopatología de la depresión. Debido al interés actual de desarrollar nuevos mecanismos antidepresivos, estos hallazgos bioquímicos sobre el metabolismo de GABA en la depresión podrían representar una alternativa a explorar para el desarrollo de nuevos tratamientos farmacológicos para la depresión.

\section{Agradecimientos}

El presente trabajo fue elaborado con el financiamiento del fondo sectorial SSA/IMSS/ISSSTE (FOSISS), Proyecto 261435.

\section{Bibliografía}

1. Nota informativa. Febrero de 2017. Disponible en: http://www.who.int/ mediacentre/factsheets/fs369/es

2. Saveanu RV, Nemeroff CB. Etiology of depression: genetic and environmental factors. Psychiatr Clin North Am. 2012;35:51-71.

3. Werner FM, Covenas R. Classical neurotransmitters and neuropeptides involved in major depression: a review. Int $\mathrm{J}$ Neurosci. 2010;120:455-70.

4. Zachmann M, Tocci P, Nyhan WL. The occurrence of gamma-aminobutyric acid in human tissues other than brain. $J$ Biol Chem. 1966;241:1355-8.

5. Xu G, Broadbelt KG, Haynes RL, et al. Late development of the GABAergic system in the human cerebral cortex and white matter. J Neuropathol Exp Neurol. 2011;70:841-58.

6. Otsuka M, Iversen LL, Hall ZW, et al. Release of gamma-aminobutyric acid from inhibitory nerves of lobster. Proc Natl Acad Sci U S A. 1966;56:1110-5.

7. Meldrum B. Pharmacology of GABA. Clin Neuropharmacol. 1982:5:293-316.

8. Guidotti A, Corda MG, Wise BC, et al. GABAergic synapses. Supramolecular organization and biochemical regulation. Neuropharmacology. 1983;22:1471-9.

9. Brambilla P, Perez J, Barale F, et al. GABAergic dysfunction in mood disorders. Mol Psychiatry. 2003;8:721-37.

10. Peng $L$, Hertz $L$, Huang $R$, et al. Utilization of glutamine and of TCA cycle constituents as precursors for transmitter glutamate and GABA. Dev Neurosci. 1993;15:367-77.

11. Scimemi A. Structure, function, and plasticity of GABA transporters. Front Cell Neurosci. 2014:8:161.

12. Schousboe A, Westergaard N, Sonnewald U, et al. Glutamate and glutamine metabolism and compartmentation in astrocytes. Dev Neurosci. 1993;15:359-66.

13. Erlander MG, Tillakaratne NJ, Feldblum S, et al. Two genes encode distinct glutamate decarboxylases. Neuron. 1991;7:91-100.

14. Wu C, Sun D. GABA receptors in brain development, function, and injury. Metab Brain Dis. 2015:30:367-79.

15. Eder $M$, Rammes $G$, Zieglgansberger $W$, et al. $G A B A(A)$ and $G A B A(B)$ receptors on neocortical neurons are differentially distributed. Eur J Neurosci. 2001;13:1065-9.

16. Costa E, Auta J, Grayson DR, et al. GABAA receptors and benzodiazepines: a role for dendritic resident subunit mRNAs. Neuropharmacology. 2002;43:925-37.

17. Kaupmann K, Malitschek B, Schuler V, et al. GABA(B)-receptor subtypes assemble into functional heteromeric complexes. Nature. 1998;396:683-7.

18. Kerr DI, Ong J. GABAB receptors. Pharmacol Ther. 1995;67:187-246.

19. Bettler B, Kaupmann K, Mosbacher J, et al. Molecular structure and physiological functions of $\operatorname{GABA}(\mathrm{B})$ receptors. Physiol Rev. 2004;84:835-67.

20. Kilb W, Kirischuk S, Luhmann HJ. Role of tonic GABAergic currents during pre- and early postnatal rodent development. Front Neural Circuits. 2013;7:139.

21. Salari AA, Bakhtiari A, Homberg JR. Activation of GABA-A receptors during postnatal brain development increases anxiety- and depression-related behaviors in a time- and dose-dependent manner in adult mice. Eur Neuropsychopharmacol. 2015;25:1260-74 
22. Hines RM, Davies PA, Moss SJ, et al. Functional regulation of GABAA receptors in nervous system pathologies. Curr Opin Neurobiol. 2012;22:552-8

23. Earnheart JC, Schweizer C, Crestani F, et al. GABAergic control of adult hippocampal neurogenesis in relation to behavior indicative of trait anxiety and depression states. J Neurosci. 2007;27:3845-54.

24. Shen Q, Lal R, Luellen BA, et al. Gamma-aminobutyric acid-type A receptor deficits cause hypothalamic-pituitary-adrenal axis hyperactivity and antidepressant drug sensitivity reminiscent of melancholic forms of depression. Biol Psychiatry. 2010;68:512-20.

25. Romeo E, Strohle A, Spalletta G, et al. Effects of antidepressant treatment on neuroactive steroids in major depression. Am J Psychiatry. 1998;155:910-3.

26. Yee BK, Keist R, von Boehmer L, et al. A schizophrenia-related sensorimotor deficit links alpha 3-containing GABAA receptors to a dopamine hyperfunction. Proc Natl Acad Sci U S A. 2005;102:17154-9.

27. Smith KS, Rudolph U. Anxiety and depression: mouse genetics and pharmacological approaches to the role of $\operatorname{GABA}(\mathrm{A})$ receptor subtypes. Neuropharmacology. 2012:62:54-62.

28. Wang JF, Sun X, Chen B, et al. Lamotrigine increases gene expression of GABA-A receptor beta3 subunit in primary cultured rat hippocampus cells. Neuropsychopharmacology. 2002;26:415-21.

29. Gassmann M, Bettler B. Regulation of neuronal $G A B A(B)$ receptor functions by subunit composition. Nat Rev Neurosci. 2012;13:380-94.

30. Frankowska M, Filip M, Przegalinski E. Effects of GABAB receptor ligands in animal tests of depression and anxiety. Pharmacol Rep. 2007;59:645-55.

31. Nakagawa $Y$, Ishima $T$, Ishibashi $Y$, et al. Involvement of GABAB receptor systems in experimental depression: baclofen but not bicuculline exacerbates helplessness in rats. Brain Res. 1996;741:240-5.

32. Heese $K$, Otten $U$, Mathivet $P$, et al. $G A B A(B)$ receptor antagonists elevate both mRNA and protein levels of the neurotrophins nerve growth factor (NGF) and brain-derived neurotrophic factor (BDNF) but not neurotrophin-3 (NT-3) in brain and spinal cord of rats. Neuropharmacology. 2000;39:449-62.

33. Nibuya M, Morinobu S, Duman RS. Regulation of BDNF and trkB mRNA in rat brain by chronic electroconvulsive seizure and antidepressant drug treatments. J Neurosci. 1995;15:7539-47.

34. Duman RS, Heninger GR, Nestler EJ. A molecular and cellular theory of depression. Arch Gen Psychiatry. 1997:54:597-606.

35. Bowery NG, Bettler B, Froestl W, et al. International Union of Pharmacology. XXXIII. Mammalian gamma-aminobutyric acid(B) receptors: structure and function. Pharmacol Rev. 2002:54:247-64.

36. Serrats J, Artigas F, Mengod G, et al. GABAB receptor mRNA in the raphe nuclei: co-expression with serotonin transporter and glutamic acid decarboxylase. J Neurochem. 2003;84:743-52.

37. Cryan JF, Kaupmann K. Don't worry 'B' happy!: a role for GABA(B) receptors in anxiety and depression. Trends Pharmacol Sci. 2005;26: 36-43.

38. Bowery NG, Hudson AL, Price GW. GABAA and GABAB receptor site distribution in the rat central nervous system. Neuroscience. 1987;20: 365-83.

39. Monteleone $\mathrm{P}$, Maj $\mathrm{M}$, lovino $\mathrm{M}$, et al GABA, depression and the mechanism of action of antidepressant drugs: a neuroendocrine approach. J Affect Disord. 1990;20:1-5.

40. Emrich HM, von Zerssen D, Kissling W, et al. Effect of sodium valproate on mania. The GABA-hypothesis of affective disorders. Arch Psychiatr Nervenkr (1970). 1980;229:1-16.

41. Gold BI, Bowers MB Jr, Roth RH, et al. GABA levels in CSF of patients with psychiatric disorders. Am J Psychiatry. 1980;137:362-4.

42. Mann JJ, Oquendo MA, Watson KT, et al. Anxiety in major depression and cerebrospinal fluid free gamma-aminobutyric acid. Depress Anxiety. 2014;31:814-21.

43. Lu YR, Fu XY, Shi LG, et al. Decreased plasma neuroactive amino acids and increased nitric oxide levels in melancholic major depressive disorder. BMC Psychiatry. 2014;14:123.

44. van Marwijk $\mathrm{H}$, Allick $\mathrm{G}$, Wegman $\mathrm{F}$, et al. Alprazolam for depression Cochrane Database Syst Rev. 2012;(7):CD007139.

45. Schatzberg AF, Cole JO. Benzodiazepines in depressive disorders. Arch Gen Psychiatry. 1978;35:1359-65.

46. Petty F, Trivedi MH, Fulton M, et al. Benzodiazepines as antidepressants does GABA play a role in depression? Biol Psychiatry. 1995;38:578-91.

47. Kucukibrahimoglu $E$, Saygin MZ, Caliskan M, et al. The change in plasma GABA, glutamine and glutamate levels in fluoxetine- or S-citalopram-treated female patients with major depression. Eur J Clin Pharmacol. 2009;65:571-7.

48. Sanacora G, Mason GF, Rothman DL, et al. Increased occipital cortex GABA concentrations in depressed patients after therapy with selective serotonin reuptake inhibitors. Am J Psychiatry. 2002;159:663-5.

49. Price RB, Shungu DC, Mao X, et al. Amino acid neurotransmitters assessed by proton magnetic resonance spectroscopy: relationship to treatment resistance in major depressive disorder. Biol Psychiatry. 2009;65:792-800.
50. Pehrson AL, Sanchez C. Altered gamma-aminobutyric acid neurotransmission in major depressive disorder: a critical review of the supporting evidence and the influence of serotonergic antidepressants. Drug Des Devel Ther. 2015;9:603-24.

51. Hasler G, van der Veen JW, Tumonis T, et al. Reduced prefrontal glutamate/glutamine and gamma-aminobutyric acid levels in major depression determined using proton magnetic resonance spectroscopy. Arch Gen Psychiatry. 2007:64:193-200.

52. Drevets WC. Functional neuroimaging studies of depression: the anatomy of melancholia. Annu Rev Med. 1998;49:341-61.

53. Sanacora G, Mason GF, Rothman DL, et al. Increased cortical GABA concentrations in depressed patients receiving ECT. Am J Psychiatry. 2003;160:577-9.

54. Bhagwagar Z, Wylezinska M, Jezzard P, et al. Low GABA concentrations in occipital cortex and anterior cingulate cortex in medication-free, recovered depressed patients. Int $\mathrm{J}$ Neuropsychopharmacol. 2008;11:255-60

55. Rajkowska G, O'Dwyer G, Teleki Z, et al. GABAergic neurons immunoreactive for calcium binding proteins are reduced in the prefrontal cortex in major depression. Neuropsychopharmacology. 2007;32:471-82.

56. Kessler RC, Ustün TB. The World Mental Health (WMH) Survey Initiative Version of the World Health Organization (WHO) Composite International Diagnostic Interview (CIDI). Int J Methods Psychiatr Res. 2004;13:93-121

57. Payne JL, Palmer JT, Joffe $\mathrm{H}$. A reproductive subtype of depression: conceptualizing models and moving toward etiology. Harv Rev Psychiatry. 2009;17:72-86

58. Flores-Ramos M. Los trastornos mentales relacionados a la edad reproductiva de la mujer: una nueva propuesta en el campo de la salud mental. Gac Med Mex. 2011;147:33-7.

59. Flores-Ramos M, Heinze G, Silvestri-Tomassoni R. Association between depressive symptoms and reproductive variables in a group of perimenopausal women attending a menopause clinic in Mexico City. Arch Womens Ment Health. 2010;13:99-105.

60. Freeman EW, Sammel MD, Lin H, et al. Associations of hormones and menopausal status with depressed mood in women with no history of depression. Arch Gen Psychiatry. 2006;63:375-82.

61. Ryan J, Burger HG, Szoeke C, et al. A prospective study of the association between endogenous hormones and depressive symptoms in postmenopausal women. Menopause. 2009;16:509-17.

62. Daly RC, Danaceau MA, Rubinow DR, et al. Concordant restoration of ovarian function and mood in perimenopausal depression. Am J Psychiatry. 2003;160:1842-6.

63. Bromberger JT, Schott LL, Kravitz HM, et al. Longitudinal change in reproductive hormones and depressive symptoms across the menopausal transition: results from the Study of Women's Health Across the Nation (SWAN). Arch Gen Psychiatry. 2010;67:598-607.

64. Flores-Ramos M, Moreno J, Heinze G, et al. Gonadal hormone levels and platelet tryptophan and serotonin concentrations in perimenopausa women with or without depressive symptoms. Gynecol Endocrinol. 2014;30:232-5.

65. Woods NF, Smith-DiJulio K, Percival DB, et al. Depressed mood during the menopausal transition and early postmenopause: observations from the Seattle Midlife Women's Health Study. Menopause. 2008;15:223-32

66. Schiller CE, Meltzer-Brody S, Rubinow DR. The role of reproductive hormones in postpartum depression. CNS Spectr. 2015;20:48-59.

67. Ben Dor R, Harsh VL, Fortinsky P, et al. Effects of pharmacologically induced hypogonadism on mood and behavior in healthy young women. Am J Psychiatry. 2013;170:426-33.

68. Fernandez-Guasti A, Fiedler JL, Herrera L, et al. Sex, stress, and mood disorders: at the intersection of adrenal and gonadal hormones. Horm Metab Res. 2012;44:607-18.

69. Goel N, Workman JL, Lee TT, et al. Sex differences in the HPA axis. Compr Physiol. 2014;4:1121-55.

70. Handa RJ, Sharma D, Uht R. A role for the androgen metabolite, 5alpha androstane 3beta, 17beta diol (3beta-diol) in the regulation of the hypothalamo-pituitary-adrenal axis. Front Endocrinol (Lausanne). 2011;2:65.

71. Handa RJ, Weiser MJ. Gonadal steroid hormones and the hypothalamo-pituitary-adrenal axis. Front Neuroendocrinol. 2014;35:197-220.

72. Walf AA, Frye CA. Estradiol reduces anxiety- and depression-like behavior of aged female mice. Physiol Behav. 2010;99:169-74.

73. Estrada-Camarena E Fernandez-Guasti A, Lopez-Rubalcava C. Antidepressant-like effect of different estrogenic compounds in the forced swimming test. Neuropsychopharmacology. 2003;28:830-8.

74. Bredemann TM, McMahon LL. 17beta estradiol increases resilience and improves hippocampal synaptic function in helpless ovariectomized rats. Psychoneuroendocrinology. 2014;42:77-88.

75. Gleason CE, Dowling NM, Wharton W, et al. Effects of hormone therapy on cognition and mood in recently postmenopausal women: findings from the randomized, controlled KEEPS-cognitive and affective study. PLoS Med. 2015;12:e1001833. 
76. Halbreich $U$, Kahn LS. Role of estrogen in the aetiology and treatment of mood disorders. CNS Drugs. 2001;15:797-817.

77. Schmidt PJ, Nieman L, Danaceau MA, et al. Estrogen replacement in perimenopause-related depression: a preliminary report. Am J Obstet Gynecol. 2000;183:414-20.

78. Ben Dor R, Marx CE, Shampine LJ, et al. DHEA metabolism to the neurosteroid androsterone: a possible mechanism of DHEA's antidepressant action. Psychopharmacology (Berl). 2015;232:3683.

79. Martinez-Mota L, Contreras CM, Saavedra M. Progesterone reduces immobility in rats forced to swim. Arch Med Res. 1999;30:286-9.

80. Rodriguez-Landa JF, Contreras CM, Bernal-Morales B, et al. Allopregnanolone reduces immobility in the forced swimming test and increases the firing rate of lateral septal neurons through actions on the GABAA receptor in the rat. J Psychopharmacol. 2007;21:76-84.

81. Schmidt PJ, Nieman LK, Danaceau MA, et al. Differential behavioral effects of gonadal steroids in women with and in those without premenstrual syndrome. N Engl J Med. 1998;338:209-16.

82. Smith MJ, Adams LF, Schmidt PJ, et al. Abnormal luteal phase excitability of the motor cortex in women with premenstrual syndrome. Biol Psychiatry. 2003;54:757-62.

83. de Souza LM, Franci CR. Differential immunoreactivity of glucocorticoid receptor and GABA in GABAergic afferents to parvocellular neurons in the paraventricular nucleus. Neurosci Lett. 2013;534:199-204.

84. Gordon JL, Girdler SS, Meltzer-Brody SE, et al. Ovarian hormone fluctuation, neurosteroids, and HPA axis dysregulation in perimenopausal depression: a novel heuristic model. Am J Psychiatry. 2015; 172:227-36.
85. Vigod SN, Strasburg K, Daskalakis ZJ, et al. Systematic review of gamma-aminobutyric-acid inhibitory deficits across the reproductive life cycle. Arch Womens Ment Health. 2014;17:87-95.

86. Mohler $\mathrm{H}$. The GABA system in anxiety and depression and its therapeutic potential. Neuropharmacology. 2012;62:42-53.

87. Loscher W. Valproate: a reappraisal of its pharmacodynamic properties and mechanisms of action. Prog Neurobiol. 1999;58:31-59.

88. Davis LL, Kabel D, Patel D, et al. Valproate as an antidepressant in major depressive disorder. Psychopharmacol Bull. 1996;32:647-52.

89. Corrigan FM. Sodium valproate augmentation of fluoxetine or fluvoxamine effects. Biol Psychiatry. 1992;31:1178-9.

90. Zarate CA Jr, Tohen M, Baraibar G. Combined valproate or carbamazepine and electroconvulsive therapy. Ann Clin Psychiatry. 1997; 9:19-25.

91. Ghabrash MF, Comai S, Tabaka J, et al. Valproate augmentation in a subgroup of patients with treatment-resistant unipolar depression. World J Biol Psychiatry. 2016;17:165-70.

92. Dubin MJ, Mao X, Banerjee S, et al. Elevated prefrontal cortex GABA in patients with major depressive disorder after TMS treatment measured with proton magnetic resonance spectroscopy. J Psychiatry Neurosci. 2016;41:150223.

93. Licata SC, Jensen JE, Conn NA, et al. Zolpidem increases GABA in depressed volunteers maintained on SSRIs. Psychiatry Res. 2014;224:28-33.

94. Zorumski CF, Paul SM, Izumi Y, et al. Neurosteroids, stress and depression: potential therapeutic opportunities. Neurosci Biobehav Rev. 2013; 37:109-22. 